\title{
Alternating commensurate-incommensurate structures in the magnetic phase diagram of $\mathrm{CsNiF}_{3}$
}

\author{
C. Pich and F. Schwabl \\ TU-München, James-Franck-Strasse, 85747 Garching, Germany
}

\begin{abstract}
The magnetic phase diagram of the quasi one-dimensional spinchain system $\mathrm{CsNiF}_{3}$ below the Néel temperature is determined. For magnetic fields perpendicular to the spin chains incommensurate phases are predicted. From linear spin-wave theory we obtain the instability line of the paramagnetic phase as a function of the strength and the direction of the field. The system undergoes a transition to a commensurate or an incommensurate phase depending on the direction of the magnetic field. In the commensurate phase the characterizing wave vector is locked to values describing a two-sublattice structure, whereas in the incommensurate phase the wave vector changes continuously between the corresponding two-sublattice wave vectors.
\end{abstract}

PACS numbers: $64.70 \mathrm{R}, 75.10 \mathrm{~J}, 75.30 \mathrm{D}, 75.50 \mathrm{E}$

Typeset Using REVTEX 
The quasi-one-dimensional magnetic compounds $\mathrm{ABX}_{3}$ (A alkaline metal, $\mathrm{B}$ transition metal, X halogen) have attracted much interest [1]:2]. These materials have ferromagnetic or antiferromagnetic intrachain interactions and weak antiferromagnetic interchain interactions in the plane. The systems with integer spin (e.g. $\mathrm{CsNiCl}_{3}$ ) are studied in context with the Haldane conjecture [3]. $\mathrm{CsFeCl}_{3}$ is discussed as an example of a system with a singletground-state [四.

$\mathrm{CsNiF}_{3}$ is an example of a ferromagnet with planar anisotropy which has been studied extensively experimentally and theoretically [4 81. It remains in the center of focus [10,11] because it is a model system with reduced dimensionality where enhanced fluctuations have a pronounced effect on the ordering structure. Due to the planar anisotropy the spins will be oriented in the plane perpendicular to the chain axis. Neutron scattering revealed the dynamics of linear and nonlinear excitations [5]. The one dimensional spin chain has no long range order but when applying a homogeneous field perpendicular to the chain axis spin waves can be measured and described within a linear spin wave theory [9]. However, the three-dimensional properties of $\mathrm{CsNiF}_{3}$ have hardly been studied. Recently Baehr et al. [10] measured the magnetic excitations in the three dimensional ordered state $\left(T<T_{N}=2.7 \mathrm{~K}\right)$. It could be shown that an isotropic antiferromagnetic exchange in the hexagonal plane and the dipole-dipole interaction are responsible for the three dimensional collinear long range order. The spins are oriented within the plane perpendicular to the chain axis along the crystal axes of the triangular lattice.

In the following we study the three dimensional magnetic structure of $\mathrm{CsNiF}_{3}$ for magnetic fields oriented in the plane. We examine the stability of the paramagnetic phase as a function of the direction of the field. By means of linear spin wave theory we obtain the instability line at which the paramagnetic spin orientation gets unstable and changes to a canted structure. It turns out that the ground state depends sensitively on the field direction. For certain angular domains $\left(\Delta \varphi_{i c} \approx 15.6^{\circ}\right)$ there exist incommensurate phases separated by commensurate phases (of width $\Delta \varphi_{c} \approx 44.4^{\circ}$ ). Thus as a function of the direction of the field we obtain alternating commensurate and incommensurate spin structures. 
This is the first time that incommensurate phases have been predicted in $\mathrm{CsNiF}_{3}$.

The starting point of our investigation is the Spin Hamiltonian

$$
H=-2 J \sum_{i} \mathbf{S}_{i} \mathbf{S}_{i+1}+A \sum_{l}\left(S_{l}^{z}\right)^{2}-\sum_{\alpha, \beta} \sum_{l, l^{\prime}}\left(J_{l l^{\prime}}^{\prime} \delta^{\alpha \beta}+A_{l l^{\prime}}^{\alpha \beta}\right) S_{l}^{\alpha} S_{l^{\prime}}^{\beta}-g_{L} \mu_{B} \mathbf{H}_{0} \sum_{l} \mathbf{S}_{l} .
$$

Here $J$ denotes the ferromagnetic nearest-neighbor intrachain interaction, $A$ the single-ion anisotropy, $J_{l l^{\prime}}^{\prime}$ the interchain and $i$ indicates positions on one and the same spin chain, whereas $l$ indicates all spin positions. $\mathbf{H}_{0}$ is an external field perpendicular to the chain axis. The magnetic lattice structure of $\mathrm{CsNiF}_{3}$ is a simple hexagonal structure with lattice constants $c=2.6 \AA$ of the spin chains and $a=6.2 \AA$ of the triangular lattice in the plane. From neutron scattering [10 the coefficients in Eq. (11) are deduced to $J=11.8 \mathrm{~K}, A=3.3$ $\mathrm{K}$ and $J^{\prime}=-0.025 \mathrm{~K}$. The value for $J^{\prime}$ is of the same order as the dipole energy which is necessary for stabilizing a collinear antiferromagnetic spin structure. Because of the large planar anisotropy the spins are forced to lie in the hexagonal plane. The classical ground state for vanishing field is given by three possible domains $\mathrm{A}-\mathrm{C}$ (s. Fig. 1) in which a collinear antiferomagnetic spin structure is realized, i.e. the rotation symmetry is broken due to the competition of the exchange and the dipole-dipole interaction [10,12]. Thus the system has an easy axis anisotropy. In the real system all three domains are simultanously present.

Fourier transformation of the Hamiltonian (Eq. (1)) yields

$$
H=-\sum_{\alpha, \beta} \sum_{\mathbf{q}}\left(J_{\mathbf{q}} \delta^{\alpha \beta}-A \delta^{\alpha z} \delta^{\beta z}+J_{\mathbf{q}}^{\prime} \delta^{\alpha \beta}+A_{\mathbf{q}}^{\alpha \beta}\right) S_{\mathbf{q}}^{\alpha} S_{-\mathbf{q}}^{\beta}-g_{L} \mu_{B} \sqrt{N} \mathbf{H}_{0} \mathbf{S}_{0}
$$

with the nearest-neighbor exchange energies (intrachain and interchain)

$$
\begin{aligned}
& J_{\mathbf{q}}=2 J \cos c q_{z} \\
& J_{\mathbf{q}}^{\prime}=2 J^{\prime}\left(\cos a q_{x}+2 \cos \frac{a q_{x}}{2} \cos \frac{\sqrt{3} a q_{y}}{2}\right) .
\end{aligned}
$$

$A_{\mathbf{q}}^{\alpha \beta}$ denotes the Fourier transform of the long-range dipole interaction, which is calculated by means of the Ewald summation technique [13, [14].

The qualitative effect of a homogeneous magnetic field is as follows. For weak magnetic fields transverse to the spin orientation the spins will reorient in order to gain energy from 
the Zeeman term. Thus when orienting the field along one domain direction the two others will change their ground state immediately. For a field longitudinal to the spins a spin-wave calculation reveals [12,15] that the Néel state is at least metastable up to a finite critical field which depends solely on the dipole energy. Thus the situation for a virgin probe neglecting effects from domain wall energies and crystal defects is as follows: Without magnetic field the system might be built up of the three domains in equal fractions. Raising the magnetic field (parallel to the spins in domain A) does not change the spin orientation in domain A but leads to a slight reorientation in domains B and C. Above the the critical value the spins in domain A flip (first order phase transition) to an orientation identical to either domain B or domain C. For strong magnetic fields one finally enters the paramagnetic phase. When the magnetic field is decreased thereafter, the spins order again in the two domains but domain $\mathrm{A}$ is not formed any more because of the metastability of this domain. The system ends up in a state, where only domains B and C are present.

In the following we study the excitations by means of linear spin wave theory. Via a Holstein-Primakoff-transformation we transform the Spin operators $S_{l}^{\alpha}$ in the Hamiltonian (Eq. 2) to Bose operators $a_{l}$ and $a_{l}^{\dagger}$ [16, [7] and diagonalize the quadratic form.

In the paramagnetic phase all spins are aligned along the magnetic field $\mathbf{H}_{0}$. Because the ground state of $\mathrm{CsNiF}_{3}$ for vanishing fields is not invariant with respect to a rotation around the spin-chain axis (recall that there are three domains $\mathrm{A}-\mathrm{C}$ ), the direction of the field plays a crucial role. The field direction is parametrized by $\varphi$, which is the angle between the field and the $x$-axis (inset in Fig. (4)). To quadratic order in the Bose operators we obtain the Hamiltonian

$$
H=E_{P M}^{c l}+\sum_{\mathbf{q}} A_{\mathbf{q}} a_{\mathbf{q}}^{\dagger} a_{\mathbf{q}}+\frac{1}{2}\left(B_{\mathbf{q}} a_{\mathbf{q}} a_{-\mathbf{q}}+B_{\mathbf{q}}^{*} a_{\mathbf{q}}^{\dagger} a_{-\mathbf{q}}^{\dagger}\right)
$$

with the coefficients

$$
\begin{aligned}
A_{\mathbf{q}}= & S\left(2\left(J_{0}-J_{\mathbf{q}}\right)+A+2 J_{0}^{\prime}-2 J_{\mathbf{q}}^{\prime}\right)+g_{L} \mu_{B} H_{0} \\
& +S\left(\cos ^{2} \varphi\left(2 A_{0}^{x x}-A_{\mathbf{q}}^{y y}\right)+\sin ^{2} \varphi\left(2 A_{0}^{y y}-A_{\mathbf{q}}^{x x}\right)-A_{\mathbf{q}}^{z z}-\sin 2 \varphi A_{\mathbf{q}}^{x y}\right) \\
B_{\mathbf{q}}= & S\left(-A+A_{\mathbf{q}}^{z z}-\sin ^{2} \varphi A_{\mathbf{q}}^{x x}-\cos ^{2} \varphi A_{\mathbf{q}}^{y y}+\sin 2 \varphi A_{\mathbf{q}}^{x y}\right)
\end{aligned}
$$


and the classical ground state energy of the paramagnetic state

$$
E_{P M}^{c l}=-N S^{2}\left(J_{0}+J_{0}^{\prime}+\left(\cos ^{2} \varphi A_{0}^{x x}+\sin ^{2} \varphi A_{0}^{y y}\right)\right)-g_{L} \mu_{B} N S H_{0}
$$

which is independent of the direction of the field for spherical shaped systems $\left(A_{0}^{x x}=A_{0}^{y y}\right)$. Here we considered only wave vectors within the plane $\left(q_{z}=0, A_{\mathbf{q}}^{y z}=0\right)$ due to the strong planar anisotropy. The dispersion relation for the paramagnetic phase is calculated via a Bogoliubov transformation to

$$
E_{\mathbf{q}}=\sqrt{A_{\mathbf{q}}^{2}-\left|B_{\mathbf{q}}\right|^{2}}
$$

This equation is valid for high magnetic fields. When lowering the magnetic field the paramagnetic phase gets unstable and changes to a canted spin structure. This instability is signalled by a soft mode at the wave vector $\mathbf{q}(\varphi)$ characterizing the phase below the paramagnetic phase. The value of the critical field $H_{0}^{c}$ and the wave vector $\mathbf{q}(\varphi)$ depend on the angle of the field $\varphi$ and can be evaluated from Eq. (8) by setting the excitation energy to zero. This leads to the equation

$$
g_{L} \mu_{B} H_{0}^{c}(\varphi)=2 S\left(J_{\mathbf{q}}^{\prime}-J_{0}^{\prime}+\sin ^{2} \varphi\left(A_{\mathbf{q}}^{x x}-A_{0}^{y y}\right)+\cos ^{2} \varphi\left(A_{\mathbf{q}}^{y y}-A_{0}^{x x}\right)-\sin 2 \varphi A_{\mathbf{q}}^{x y}\right) .
$$

Note that this expression is independent of the ferromagnetic exchange and the anisotropy energies as long as they are much larger than the antiferromagnetic exchange and the dipole energies. Regarded as a function of $\mathbf{q}$ the maximum of this expression gives the angular dependent critical value $H_{0}^{c}(\varphi)$ and the wave vector $\mathbf{q}(\varphi)$. Assuming that the phase joining the paramagnetic is a conventional spin-flop phase $(\alpha=\beta$ in inset of Fig. (4)) or a general two-sublattice structure, the so-called intermediate phase $(\alpha \neq \beta$ in inset of Fig. (4)), we expect the maximum value for the critical field at wave vectors describing the antiferromagnetic domains, i.e. $\mathbf{q}_{1}=\frac{2 \pi}{\sqrt{3} a}(0,1,0), \mathbf{q}_{2}=\frac{\pi}{a}(1,1 / \sqrt{3}, 0)$ or $\mathbf{q}_{3}=\frac{\pi}{a}(1,-1 / \sqrt{3}, 0)$ (s. Fig. 2). However, the detailed analysis shows that the paramagnetic phase gets unstable at an incommensurate wave vectorq $(\varphi)$ for certain field directions. Before considering the general case we study the special angles $\varphi=0^{\circ}$ and $\varphi=90^{\circ}$. 
(i) $\varphi=90^{\circ}$ : For magnetic fields parallel to the $y$-axis the critical value is given by

$$
g_{L} \mu_{B} H_{0}^{c y}=2 S\left(J_{\mathbf{q}}^{\prime}-J_{0}^{\prime}-A_{0}^{y y}+A_{\mathbf{q}}^{x x}\right) .
$$

This expression is found to have its maximum value of at $\mathbf{q}_{1}$, the antiferromagnetic wave vector of the collinear phase of domain A. The actual value for the critical field is evaluated with the parameters given above to (spherical shape)

$$
H_{0}^{c y}=340 \mathrm{mT} \quad \text { at } \quad \mathbf{q}\left(90^{\circ}\right)=\mathbf{q}_{1} .
$$

At $H_{0}^{c y}$ the system undergoes a transition into a commensurate phase, precisely to the two sublattice phase described by $\mathbf{q}_{1}$. Owing to the hexagonal symmetry, the critical field for $\varphi=30^{\circ}$ is the same as for $\varphi=90^{\circ}$ but at the antiferromagnetic wave vector $\mathbf{q}_{2}$ characterizing domain C; for $\varphi=-30^{\circ}$ the structure is given by $\mathbf{q}_{3}$ corresponding to domain B (s. Fig. $(5))$.

(ii) $\varphi=0^{\circ}$ : For this field direction Eq. (9) reduces to:

$$
g_{L} \mu_{B} H_{0}^{c x}=2 S\left(J_{\mathbf{q}}^{\prime}-J_{0}^{\prime}-A_{0}^{x x}+A_{\mathbf{q}}^{y y}\right) .
$$

The maximum value is not achieved for any of the two-sublattice wave vectors but for a wave vector with only an $\mathbf{q}_{x}$-component depending on the relative strength of the antiferromagnetic exchange and the dipole energy. This follows from the fact that the dipole component $A_{\mathbf{q}}^{y y}$ has a linear wave vector dependence at $\mathbf{q}_{0}$ rather than a quadratic as found for $J_{\mathbf{q}}[18$. Evaluation of the critical value for $\mathrm{CsNiF}_{3}$ leads to (spherical shape)

$$
H_{0}^{c x}=290 \mathrm{mT} \quad \text { at } \quad \mathbf{q}(0)=\frac{\pi}{a}(1.023,0,0) .
$$

Thus the system undergoes a transition to an incommensurate phase. The incommensurate wave vector happens to be near the wave vector $\mathbf{q}_{4}=\frac{\pi}{a}(1,0,0)$, characterizing a foursublattice structure, which describes an antiferromagnetic modulation along the $x$-axis.

(iii) arbitrary $\varphi=$ : Finally we turn to arbitrary angles, for which the situation turns out to be nontrivial. The complete dependence on the field direction $\mathbf{q}(\varphi)$ for $\mathrm{CsNiF}_{3}$ is 
plotted in Fig. (3). The wave vector components $q_{x}$ and $q_{y}$ are plotted as a function of the angle $\varphi$ for the region in question. A critical angle $\varphi_{c} \approx 7.8^{\circ}$ is obtained above which the wave vector is locked to $\mathbf{q}_{2}$. Varying the angle $\varphi$ between $0^{\circ}$ and $7.8^{\circ}$ the wave vector

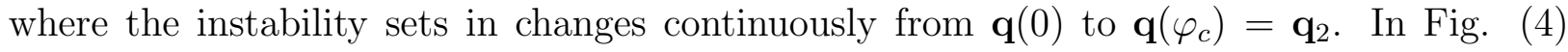
the critical value for the magnitude of the magnetic field is plotted as a function of the angle for the same angular domain. Note that the critical field is continuous even at the critical angle $\varphi_{c}$. It has only a small kink at this point. The dashed curve results from Eq. (9) under the assumption that the instability point occurs at $\mathbf{q}_{2}$ for the whole angular segment. Herefrom we can see that for $\varphi<\varphi_{c}$ the incommensurate structure is favored. Due to the inversion symmetry of the lattice together with $\mathbf{q}(\varphi)$ there is a second modulation wave vector $-\mathbf{q}(\varphi)$. The result of this investigation for all angles $\varphi$ of the magnetic field is summarized in Fig. (5): The instability line of the paramagnetic phase is shown. There are angular regions where the paramagnetic structure undergoes a transition to a two-sublattice structure (annotated by the corresponding wave vector) seperated by regions drawn in thick where an incommensurate structure is formed.

Examination of the commensurate phase leads to the following results: The commensurate phases are described near the transition to the paramagnetic phase by a two-sublattice structure. Calculation of the classical ground state energy [12 shows that a conventional spin-flop phase which is parametrized by a single angle for both sublattice spins is stable only for the field directions of $\varphi=30^{\circ}+n 60^{\circ}$ and integer values of $n$. For all other field directions the commensurate phase is an intermediate phase with two independent angles.

In summary we have studied the ground state for $\mathrm{CsNiF}_{3}$ with a homogeneous magnetic field oriented in the hexagonal plane. By stability investigations of the paramagnetic phase we obtained a non-circular instability line for fields in the hexagonal plane. The magnitude of the critical field and the type of phase joining the paramagnetic phase depends crucially on its direction. For certain angular domains the system changes to incommensurate structures, which are seperated by commensurate (two-sublattice) ones (Fig. (5)). This is the first time a staircase like behavior is found in a magnetic system as a function of the direction 
of the field. The number of steps is 6, which corresponds to twice the number of twosublattice wave vectors. This staircase is quite different from a devil's staircase with an infinite number of lock-in steps $[19,20]$. Our results for $\mathrm{CsNiF}_{3}$ are in contrast to Yamazaki et al. 81, who predicted conventional spin-flop phases for all field directions. This may result from their semiclassical model which does not consider the full nature of the dipoledipole interaction. According to our theory the magnetic phase diagram shows a much richer structure including intermediate spin configurations and incommensurate phases. The observation of our predicted new phases and the wave vector dependence is left to future experiments, e.g. neutron scattering.

\section{ACKNOWLEDGMENTS}

This work has been supported by the German Federal Ministry for Education and Research (BMBF) under the contract number 03-SC4TUM. 


\section{REFERENCES}

[1] B. Schmid, B. Dorner, D. Petitgrand, P.L. Regnault and M. Steiner, Z. Phys. B 95, 13 (1994); P. Lindgård, B. Schmid, Phys. Rev. B48, 13636 (1993).

[2] B.D. Gaulin, in Magnetic systems with competing interactions, edited by H.T. Diep, World Scientific (1994).

[3] F.D.M. Haldane, Phys. Rev. Lett. 50, 1153 (1983); T. Inam, K. Kakurai, H. Tanaka, M. Enderle and M. Steiner, J. Phys. Soc. Jpn. 63, 1530 (1995).

[4] M. Steiner, J. Villain and C.G. Windsor, Adv. Phys. 25, 87 (1976).

[5] M. Steiner M.J. Mikeska, Adv. Phys 40, 191 (1991).

[6] M. Steiner, B. Dorner, Solid State Commun. 12, 537 (1973); M. Steiner, H. Dachs, Solid State Commun. 14, 841 (1974).

[7] H. Shiba, N. Suzuki, J. Phys. Soc. Jpn. 51, 3488 (1982).

[8] H. Yamazaki, E. Soares, H. Panepucci and Y. Morishige, J. Phys. Soc. Jpn. 47, 1464 (1979) and J. Phys. Soc. Jpn. 48, 1453 (1980)

[9] M. Steiner, J. K. Kjems, J. Phys. C: Solid State Phys., 10, 2665 (1977).

[10] M. Baehr, M. Winkelmann, P. Vorderwisch, M. Steiner, C. Pich, F. Schwabl, accepted for publication in Phys. Rev. B.

[11] L.S. Campana, A. Caramico D'Auria, F. Esposito, U. Esposito and G. Kamieniarz, Phys. Rev. B 53, 2594 (1996).

[12] C. Pich and F. Schwabl, in preparation.

[13] L. Bonsal and A.A. Maradudin, Phys. Rev. B 15, 1959 (1977).

[14] M.H. Cohen, F. Keffer, Phys. Rev. 99, 1135 (1955).

[15] C. Pich and F. Schwabl, Phys. Rev. B 47, 7957 (1993). 
[16] J.M. Ziman, Principles of the theory of Solids, (Cambridge Press, 1969), p. 317ff

[17] F. Keffer, in Encyclopedea of Physics, Vol. XVIII/2, edited by S. Flügge (SpringerVerlag, Heidelberg, 1966), p. 37ff

[18] H. Shiba, Solid State Commun. 41, 511 (1982).

[19] P. Bak, Rep. Prog. Phys. 45, 587 (1982).

[20] W. Selke, in Phase Transitions and Critical Phenomenona, Vol. 15, eds. C. Domb and J.L. Lebowitz (Academic Press, New York, 1993). 


\section{FIGURES}

FIG. 1. The ground state for $\mathrm{CsNiF}_{3}$ in the hexagonal plane is one of the three shown configurations $(\mathrm{A}-\mathrm{C})$, called domains. In domain $\mathrm{A}$ the two primitive vectors are represented. The antiferromagnetic modulation can be described by $\mathbf{q}_{1}, \mathbf{q}_{2}$ and $\mathbf{q}_{3}$ for domain $\mathrm{A}, \mathrm{B}$ and $\mathrm{C}$ respectively.

FIG. 2. The Brillouin zones of the hexagonal plane. The hexagon is the crystallographic and the rectangle (dashed) the magnetic one.

FIG. 3. Wave vector $\left(q_{x}, q_{y}\right)$ at which the paramagnetic phase gets unstable versus the direction of the external magnetic field (solid line: $q_{y}$, dashed line: $q_{x}$ ). Parameters for $\mathrm{CsNiF}_{3}$ are used. For $\varphi>7.8^{\circ}$ the instability appears for $\mathbf{q}_{2}$.

FIG. 4. Critical field below which the paramagnetic phase gets unstable for field direction between $0^{\circ}$ and $30^{\circ}$. For smaller (larger) angles the paramagnetic phase changes to an incommensurate (commensurate) phase. The dashed curve indicates the critical field when assuming that the soft mode occurs at $\mathbf{q}_{2}$. The inset shows the coordinate system for a general two-sublattice spin orientation.

FIG. 5. Angular dependence of the instability of the paramagnetic phase for $\mathrm{CsNiF}_{3}$. The thick segments on the instability curve correspond to directions of the magnetic field $\mathbf{H}_{0}=\mathrm{H}_{0}(\cos \varphi, \sin \varphi, 0)$ for which the paramagnetic phase changes to an incommensurate phase. These alternate with segments where a transition to a commensurate structure appears. The corresponding wave vector is given. 


$$
\begin{aligned}
& \text { (A) } \leftarrow \leftarrow \leftarrow \leftarrow \vee \vee \wedge \vee 1 \\
& \rightarrow \rightarrow \overrightarrow{\mathrm{b}} \rightarrow \wedge \vee / \vee \\
& \leftarrow \sqrt[a]{\leftarrow} \leftarrow<\vee \vee \wedge / \vee 1 \\
& \rightarrow \rightarrow \rightarrow \rightarrow \vee 1 \vee 1 \vee
\end{aligned}
$$

י⿳亠口冖!

$$
\begin{aligned}
& \text { C } \downarrow \uparrow \downarrow \uparrow \\
& \checkmark \uparrow \downarrow 1 \\
& \downarrow \uparrow \downarrow \uparrow \\
& \downarrow \uparrow \vee 1
\end{aligned}
$$




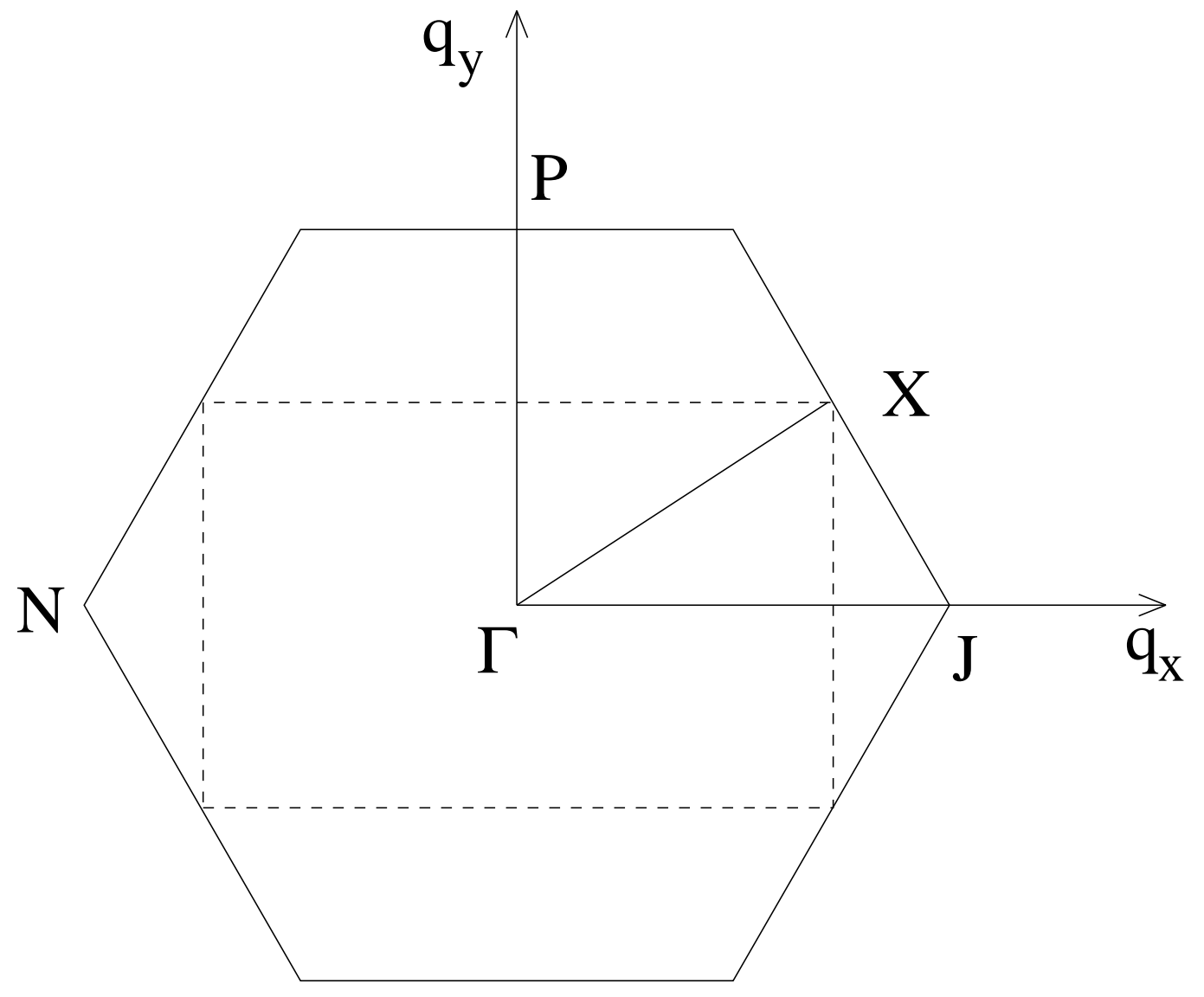

Fig. 2 


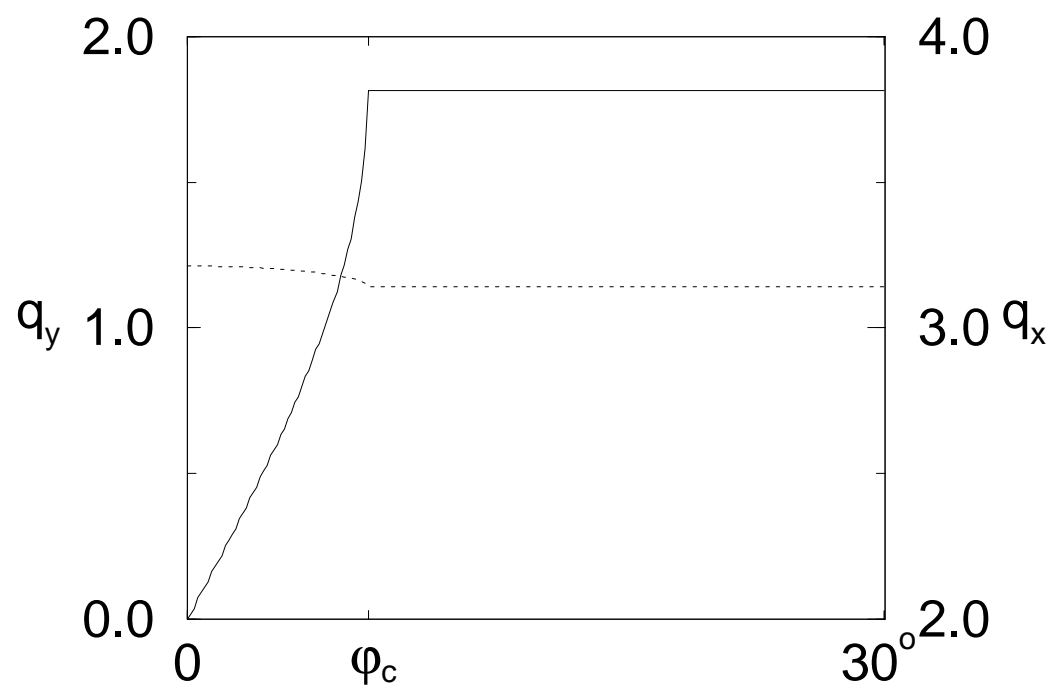

Fig. 3 


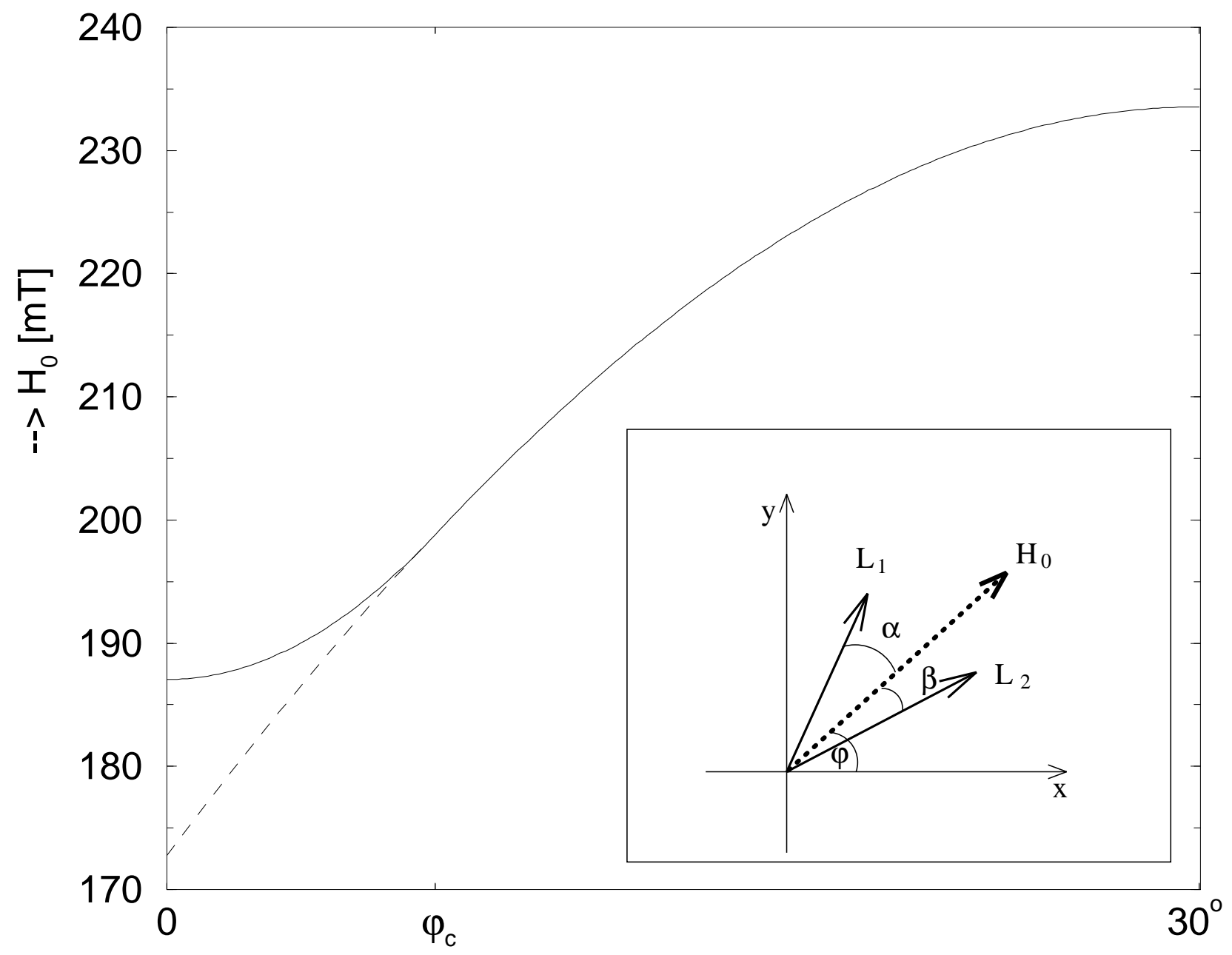

Fig. 4 


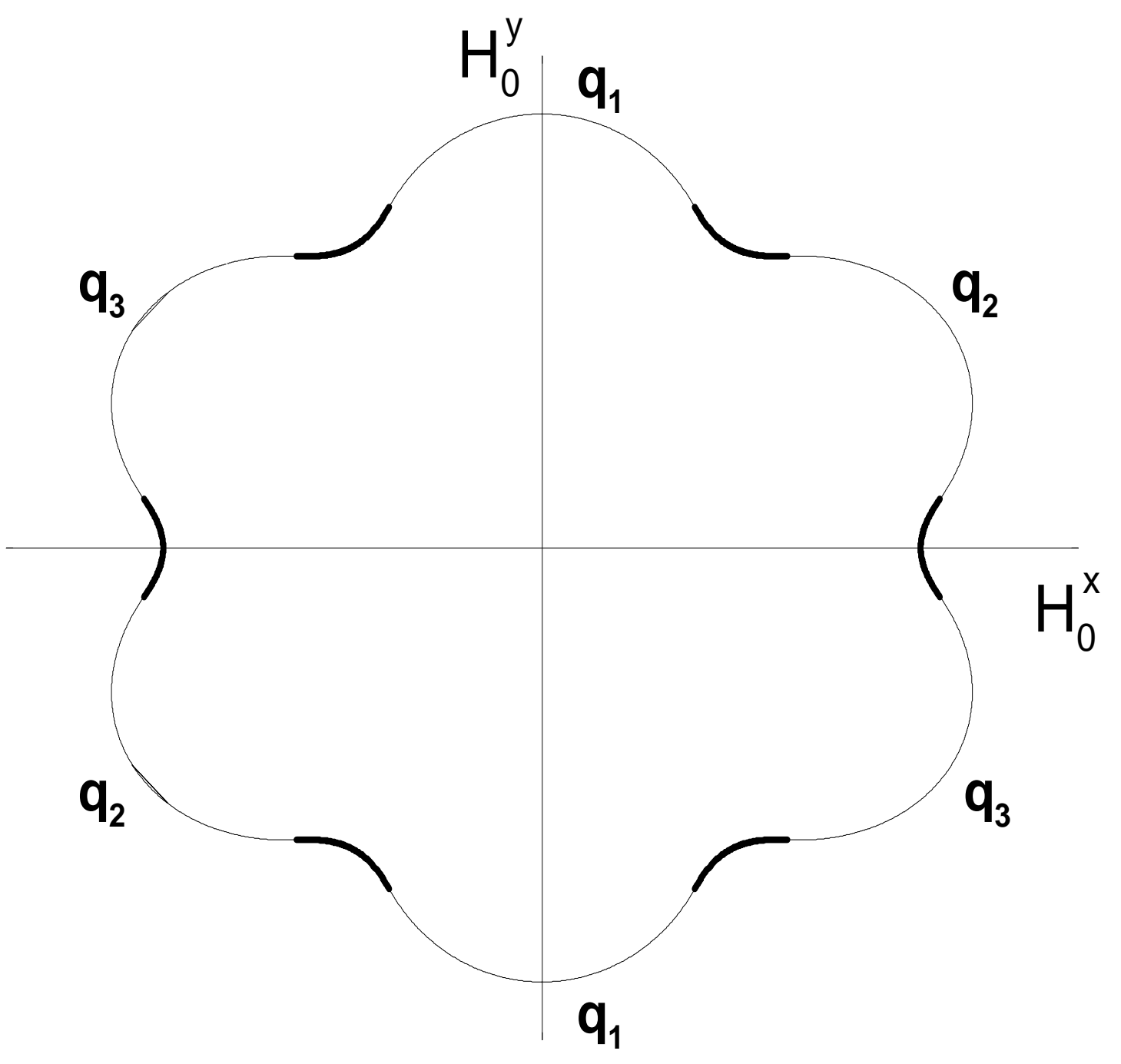

Fig. 5 